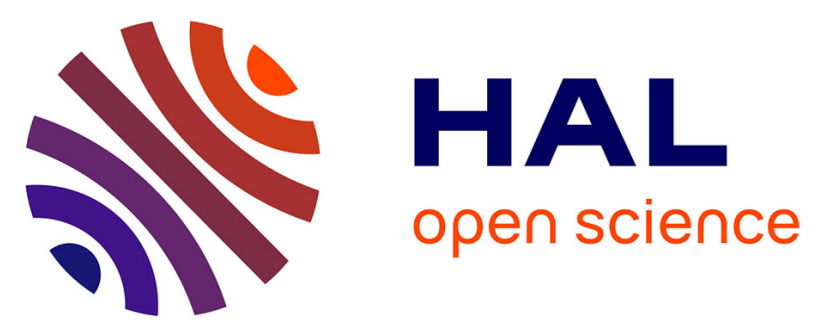

\title{
L'évolution des falaises argilo-crayeuses et limoneuses du nord du Boulonnais (Strouanne, Sangatte, France) / Evolution of the clay-chalk and silt cliffs in northern Boulonnais (Strouanne, Sangatte, France)
}

Guillaume Pierre, Philippe Lahousse

\section{To cite this version:}

Guillaume Pierre, Philippe Lahousse. L'évolution des falaises argilo-crayeuses et limoneuses du nord du Boulonnais (Strouanne, Sangatte, France) / Evolution of the clay-chalk and silt cliffs in northern Boulonnais (Strouanne, Sangatte, France). Géomorphologie: relief, processus, environnement, 2004, 10, pp.211 - 223. 10.3406/morfo.2004.1219 . hal-03277106

\section{HAL Id: hal-03277106 \\ https://hal.science/hal-03277106}

Submitted on 2 Jul 2021

HAL is a multi-disciplinary open access archive for the deposit and dissemination of scientific research documents, whether they are published or not. The documents may come from teaching and research institutions in France or abroad, or from public or private research centers.
L'archive ouverte pluridisciplinaire HAL, est destinée au dépôt et à la diffusion de documents scientifiques de niveau recherche, publiés ou non, émanant des établissements d'enseignement et de recherche français ou étrangers, des laboratoires publics ou privés. 


\section{L'évolution des falaises argilo-crayeuses et limoneuses du nord du Boulonnais (Strouanne, Sangatte, France) / Evolution of the clay-chalk and silt cliffs in northern Boulonnais (Strouanne,} Sangatte, France)

In: Géomorphologie : relief, processus, environnement. Juillet-septembre, vol. 10, n³. pp. 211-223.

\section{Résumé}

Résumé La valeur et les modalités du recul de la falaise de Strouanne qui évolue par coulées boueuses et par tassement de pans crayeux, et de celle de Sangatte, où dominent ravinements et éboulements, ont été analysées à très grande échelle et sur deux pas de temps, 150 ans pour la méthode cadastrale et 60 ans pour la méthode photogrammétrique. La variabilité spatiale de leur évolution trouve une explication dans la structure (rapport argile du Gault/craie bleue cénomanienne à Strouanne et granulométrie des lits des dépôts de versant pleistocenes à Sangatte) et dans la topographie de l'arrière de la falaise. Les deux paramètres conditionnent le bilan infiltration-ruissellement des eaux continentales. La cartographie détaillée, à deux ans d'intervalle, des mouvements de terrain qui affectent la falaise de Strouanne, et le suivi d'une ravine à Sangatte permettent de préciser, sur un pas de temps très court, l'action des processus continentaux.

\section{Abstract}

Abstract Cliff retreat in the Gault clay and chalk at Strouanne and in the Pleistocene slope deposits at Sangatte is the result of landslides at the first site and gullying and debris falls at the second. The evolution of both cliffs has been studied in detail and over a period spanning 150 and 60 years, respectively, by using cadastral surveys and stereophotogrammetry. The spatial distribution of the distance and modes of retreat allows us to highlight the significant influence of structural and topographic parameters through the way influence the balance between runoff and infiltration. During two years, comparison of digital maps with the landslides at Strouanne and monitoring of a gully at Sangatte have helped us to characterise the subaerial processes involved in cliff recession.

Citer ce document / Cite this document :

Pierre Guillaume, Lahousse Philippe. L'évolution des falaises argilo-crayeuses et limoneuses du nord du Boulonnais (Strouanne, Sangatte, France) / Evolution of the clay-chalk and silt cliffs in northern Boulonnais (Strouanne, Sangatte, France). In: Géomorphologie : relief, processus, environnement. Juillet-septembre, vol. 10, n³. pp. 211-223.

doi : $10.3406 /$ morfo.2004.1219

http://www.persee.fr/web/revues/home/prescript/article/morfo_1266-5304_2004_num_10_3_1219

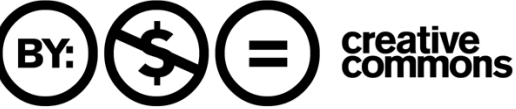




\title{
L'évolution des falaises argilo-crayeuses et limoneuses du nord du Boulonnais (Strouanne, Sangatte, France) Evolution of the clay-chalk and silt cliffs in northern Boulonnais (Strouanne, Sangatte, France)
}

\author{
Guillaume Pierre*, Philippe Lahousse*
}

\begin{abstract}
Résumé
La valeur et les modalités du recul de la falaise de Strouanne qui évolue par coulées boueuses et par tassement de pans craycux, et de celle de Sangatte, où dominent ravinements et éboulements, ont été analysées à très grande échelle et sur deux pas de temps, 150 ans pour la méthode cadastrale et 60 ans pour la méthode photogrammétrique. La variabilité spatiale de leur évolution trouve une explication dans la structure (rapport argile du Gault/craie bleue cénomanienne à Strouanne et granulométrie des lits des dépôts de versant pléistocènes à Sangatte) et dans la topographie de l'arrière de la falaise. Les deux paramètres conditionnent le bilan infiltration-ruissellement des caux continentales. La cartographie détaillée, à deux ans d'intervalle, des mouvements de terrain qui affectent la falaise de Strouanne, et le suivi d'une ravine à Sangatte permettent de préciser, sur un pas de temps très court, l'action des processus continentaux.
\end{abstract}

Mots clés : recul des falaises, photogrammétrie, processus, argile du Gault, limons pléistocènes, Boulonnais.

\begin{abstract}
Cliff retreat in the Gault clay and chalk at Strouanne and in the Pleistocene slope deposits at Sangatte is the result of landslides at the first site and gullying and debris falls at the second. The evolution of both cliffs has been studied in detail and over a period spanning 150 and 60 years, respectively, by using cadastral surveys and stereophotogrammetry. The spatial distribution of the distance and modes of retreat allows us to highlight the significant influence of structural and topographic parameters through the way these influence the balance between runoff and infiltration. During two years, comparison of digital maps with the landslides at Strouanne and monitoring of a gully at Sangatte have helped us to characterise the subaerial processes involved in cliff recession.
\end{abstract}

Key words: cliff retreat, photogrammetry, process, Gault clay, Pleistocene sill, Boulonnais.

\section{Abridged English Version}

The cap Blanc-Nez site (fig. 1) corresponds to the northern edge of the Artois under-block rising above the Flanders plain and the Straits of Dover (Colbeaux et al., 1980). In its southern part, the Strouanne cliff is cut from bottom to top in the Gault clay (Albian) and the blue chalk of the lower Cenomanian (fig. 2, Robaszynski and Amédro, 1993), and as such is similar to the cliffs of southern England described by Hutchinson (1969, 1983). Northward, Pleistocene slope deposits crop out along the Sangatte cliff (figs. 3 and 4, Sommé et al., 1999). In conformity with the dip directions of the stratified deposits that are located in the unsaturated zone of the aquifer (Bracq et al., 1992; Bracq and Brunin, 1999), percolation water flows towards the NNE. At Strouanne, the low permeability of the blue chalk results in surface runoff, channelled down to the sea by the "cran" Saint-Pô. Spring tides can reach an elevation of $4 m$ NGF. At this level the cliff base is not undercut by waves during fair weather conditions.

Cliff retreat was measured using cadastral surveys and maps at the 1/2,000 scale obtained by photogrammetric restitution (figs. 5, 6, and 8). At Strouanne, the mean cliff retreat rate between 1939 and 2002 was $0.20 \mathrm{~m} \cdot \mathrm{yr}^{\prime \prime}$ with minimum and maximum values of 0 and $0.63 \mathrm{~m} \cdot \mathrm{yr}^{-1}$ (fig. 5). The highest value corresponds to the "cran" Saint-Pô, where the cliff is $23 \mathrm{~m}$ high. Meanwhile the retreat at the Saint-Pô head $140 \mathrm{~m}$ high) has only been 6 to $15 \mathrm{~m}$. At Sangatte, from 1944 to 2002 , the mean annual retreat rate was $0.29 \mathrm{~m} \cdot \mathrm{yr}^{\prime}$, a somewhat lower retreat rate having been measured between the ancient German cemetery and the slight elevation $(29 \mathrm{~m})$ eastward of this location $\left(0.22 \mathrm{~m} \cdot \mathrm{r}^{\prime}\right)$ (fig. 6). Over approxi-

\footnotetext{
* Laboratoire de Géomorphologie et Gestion des Milieux Naturels. UFR de Géographie et Aménagement. université de Sciences et Technologies de Lille. Av. Paul Langevin, 596555 Villeneuve d'Ascy cedex. France. E-mail : guillaume pierre(a univ-lille $1 . \mathrm{fr}$
} 
mately 60 years, these cliffs have experienced continuous retreat achieved by high frequency, low-to-medium magnitude events (fig. 7, tab. 1), occasional and massives failures being controlled by the geological structure and the height of the cliff face. Along the cliff at Strouanne, comparison between digital topographic maps produced from tacheometer surveys made during spring 2001 and 2003 showed that subaerial processes play a major role in the evolution of the mud flows and the collapse structures (fig. 9).

Mud flows at cran Saint-Pô can be the result of creeping of the Gault clay as well as of the flow of the weathered chalk and overlying superficial deposits, depending on the amount of rainfall. Southward, planar slides occur where the chalk outcrop is both thin and solid with loose slabs at the top of the cliff. The bedded deposits of the Sangatte cliff are eroded as illustrated on figure 10. Runoff water on the cliff backslope percolates through open cracks and flows out on the cliff face creating tapping holes and gullying which

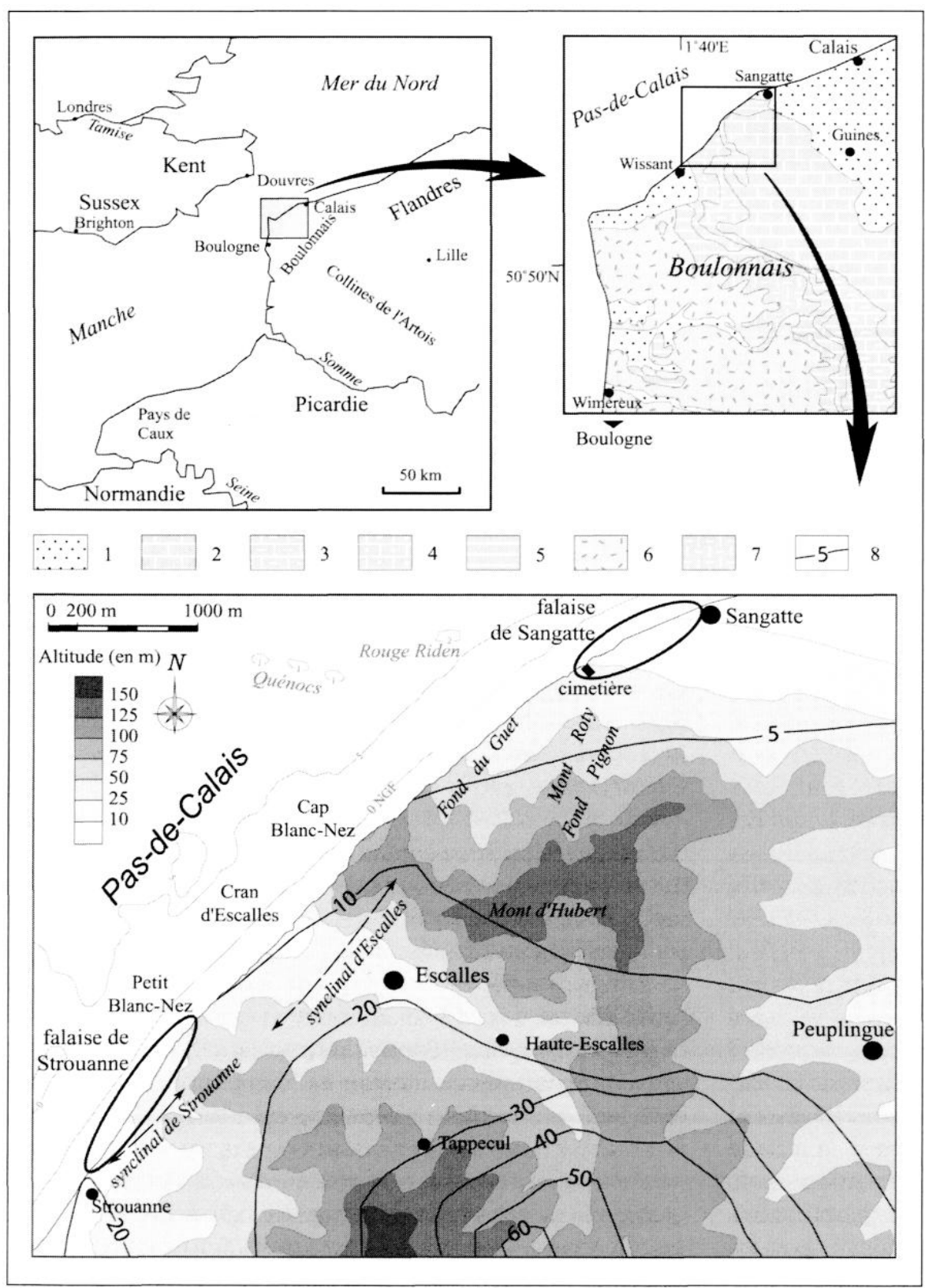

finally reaches the top of the cliff face. This results in dissecting the cliff face. The gullying eventually causes debris falls, in addition to desquamation and small liquid landfalls. There again, subaerial processes are more efficient than marine processes.

The spatial distribution of the amount and modes of retreat allows us to point out the significant influence of structural and topographical factors through the way they influence the balance between runoff and infiltration, and at places, the weathering of the chalk (cran Saint-Pô). The mass movements affecting the cliff of Strouanne are also determined by the ratio between Gault clay and chalk, the chalk contributing to slow down retreat rates. At Sangatte, structure and topography on the cliff backslope act in the same way. Eastward of Mont Roty (fig. 1), runoff water flowing down from le Fond Pignon infiltrates into the Pleistocene deposits before emerging on the cliff face. Westward, the ancient German cemetery is located too far downstream in the Fond du Guet channel and is not affected by the combination of infiltration with tapping and gullying. Because the values of cliff retreat over 150 years (1834-1987) in one case and 60 years $(1939$ or 1944-2002) in the other are nearly identical, it is probable that the retreat rate has increased since 1987. This may be related to an observed increase in rainfall (Pierre and Lahousse, 2003).

\section{Introduction}

Les falaises du Boulonnais, qui présentent une large gamme de formes et sont taillées dans des matériaux de nature et de disposition variables, n'ont pas toutes suscité le même intérêt. Après la synthèse de Briquet (1930), divers auteurs sont revenus sur les

Fig. 1 - Localisation et hydrogéologie du site. 1 : Quaternaire ; 2 : Sénonien et Turonien supérieur (craie blanche à silex) ; 3 : Turonien moyen et inférieur (craie noduleuse et marnes) ; 4 : Cénomanien (craie marneuse) ; 5 : Crétacé inférieur (argile et sables) ; 6 : Jurassique de la boutonnière du Boulonnais (grès, argiles et calcaires marneux) ; 7 : Primaire (calcaires, dolomies et schistes). 8 : courbes piézométriques (altitudes en $m$ NGF).

Fig. 1 - Location map and hydrogeological parameters. 1: Quaternary; 2: Senonian and upper Turonian (white chalk with flints); 3: Middle and lower Turonian (nodular chalk and marl); 4: Cenomanian (marly chalk); 5: Lower Cretaceous (clay and sand); 6: Jurassic (sandstone, clay and marly limestone); 7: Palaeozoic (limestone, dolomite and schist). 8: piezometric curves (elevations in metres NGF). 
falaises gréseuses (Jurassique) comprises entre Boulogne et le cap Gris-Nez (Leroux, 1959 ; Debrabant et al., 1994 ; Battiau et al., 2003), ainsi que sur les falaises crayeuses (Cénomanien) du cap Blanc-Nez (Lahousse et al., 2000 ; Lahousse et Pierre. 2002, 2003a et b). La falaise de Strouanne taillée dans l'argile albienne et la craie cénomanienne, et celle de Sangatte qui recoupe des dépôts de versants pléistocènes. n'ont en revanche jamais été l'objet de recherches concernant leur évolution, quel qu'en soit le pas de temps (fig. 1).

La première s'apparente aux falaises des côtes sud de l'Angleterre taillées dans les mêmes étages (Osmann, 1917 ; Wood, 1955 ; Brunsden et Jones, 1976), qui ont fait l'objet d'approches centrées sur la géotechnique (Hutchinson, 1969) ou sur les paramètres structuraux (Hutchinson et al., 1980 ; Hutchinson, 1983). La seconde, taillée dans des dépôts lités de versant, ne trouve pas d'équivalent strict compte tenu de la grande diversité de ce type de formations. On peut citer, néanmoins, l'étude des paramètres contrôlant l'évolution des falaises recoupant les sédiments détritiques meubles miocènes à quaternaires de l'Algarve (Alveirinho Dias et Neal, 1992), ou encore l'étude des falaises du Pays de Galles entaillant till et formations à blocs (Jones et Williams, 1991).

Outre que les conditions d'évolution de falaises d'un même type ne peuvent être similaires, tant les paramètres à prendre en compte sont variés, la plupart des exemples cités ci-dessus ne s'appuie pas sur des mesures précise du recul des falaises. Dans le nord du Boulonnais, la possibilité de travailler à très grande échelle a permis d'analyser en détail la variabilité spatiale des processus ainsi que celle du rythme et des valeurs de recul. Le rapprochement entre ces trois données et les paramètres hydrologiques (marins et continentaux), structuraux et topographiques propres au site étudié, permet d'aborder, sans a priori, la question du rôle respectif des processus marins et des processus continentaux dans l'évolution de ces falaises.

\section{Contexte structural et hydrologique}

Le site du Blanc-Nez, terminaison septentrionale du Boulonnais, correspond au rebord nord du sousbloc Artois soulevé au-dessus du Bas-Pays et du détroit du Pas-de-Calais (Colbeaux et al., 1980). La haute falaise crayeuse cénomano-turonienne du secteur Petit Blanc-Nez $(50 \mathrm{~m})$ Blanc-Nez $(100 \mathrm{~m})$ est prolongée, vers le nord et le sud. par des falaises moins hautes et de structure différente. Au sud du Petit Blanc-Nez $(50 \mathrm{~m})$, la falaise de Strouanne s'abaisse vers le hameau du même nom pour atteindre une hauteur de $15 \mathrm{~m}$. Elle est taillée à sa base dans l'argile du Gault (Albien) et à son sommet dans la craie bleue du Cénomanien inférieur (fig. 2 ; Robaszynski et Amédro, 1993). Au nord du Blanc-Nez (fig. 1), à partir de l'ancien cimetière allemand ( $28 \mathrm{~m}$ ). la falaise crayeuse. qui prend une orientation ouest- est, est fossilisée par des dépôts pléistocènes taillés en falaise jusqu'à la digue de Sangatte $(14 \mathrm{~m})$. Il s'agit d'un ensemble dont la base est composée de dépôts marins (cordons de galets et plage sableuse), recouverts au pied de la falaise fossile par des éboulis calcaires, et, en s'en éloignant, par des formations de versant argileuses, limoneuses ou sableuses dont les faciès sont décrits sur les figures 3 et 4 (Sommé et al., 1999).

La formation de Sangatte est intégralement comprise dans la zone non saturée surplombant l'aquifère crayeux (Bracq et al., 1992 ; Bracq et Brunin, 1999), et les percolations s'y font vers le nord-nord-est en vertu de la stratification et du pendage des dépôts. Entre le Petit Blanc-Nez et Strouanne, malgré la présence d'une ondulation synclinale parallèle à la côte (Destombes et Destombes, 1963 ; fig. 1), la nappe de la craie disparaît en même temps que la craie grise du Cénomanien moyen. L'affleurement de la craic bleue, faiblement perméable, induit un ruissellement de surface, canalisé vers la mer par le cran Saint-Pô (fig. 5). À Strouanne où l'épaisseur de la craie bleue est minimale, l'infiltration, très réduite, détermine la présence de sources au contact argile-craie.

Sur le site, le marnage est de $7 \mathrm{~m}$ pour un coefficient de 111 (le plus fort coefficient de pleine mer de vive eau pour l'année 2003). L'estran est alors découvert sur $750 \mathrm{~m}$ à Strouanne et $450 \mathrm{~m}$ à Sangatte. Pour ce même coefficient, la marée haute ne dépasse pas la cote 4,23 m NGF et n'atteint donc pas le pied de la falaise par temps calme (fig. 5 et fig. 6). Vers Strouanne, une haute mer de fort coefficient (>90) se tient entre $1 \mathrm{~m}$ et $30 \mathrm{~m}$ du pied de la falaise, et vers

Fig. 2 - Lithostratigraphie de la falaise de Strouanne (d'après Robaszynski et Amédro, 1993, modifié).

Fig. 2 - Lithostratigraphy of Strouanne cliff (after Robaszynski et Amédro, 1993, modified).

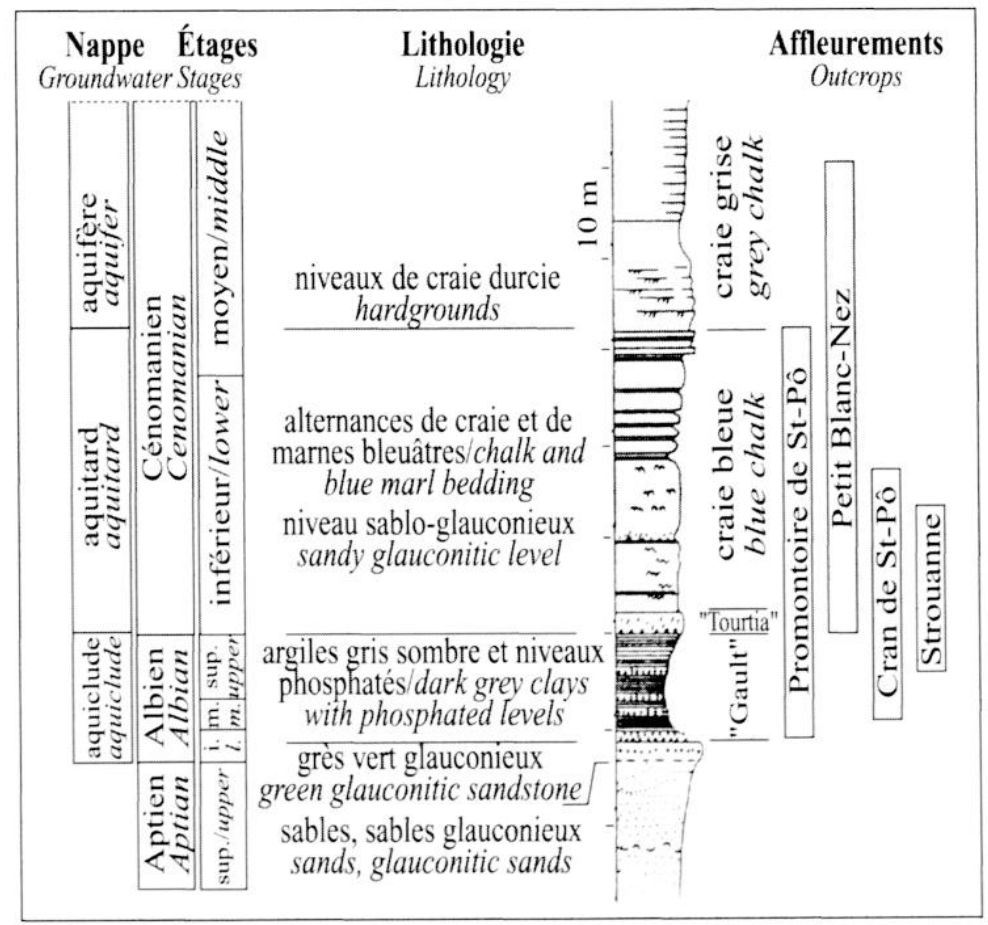


Sangatte, en fonction de la largeur, variable, du cordon de galets, le trait de côte reste une dizaine de mètres en avant du pied de la falaise.

\section{Méthodes}

Les valeurs de recul ont été établies, comme pour les falaises crayeuses du cap Blanc-Nez (Pierre et Lahousse, 2003), selon deux méthodes : comparaison de plans cadastraux et comparaison de plans topographiques établis par procédés photogrammétriques. De nombreux auteurs ont eu recours à la comparaison des cadastres napoléoniens avec ceux d'éditions plus récentes pour estimer la vitesse de recul du trait de côte (Prêcheur, 1960 ; Maquaire et al., 1984 ; Clique et Lepetit, 1986 ; Maquaire, 1990 ; Wissocq, 1992 ; Albinet, 2000 ; Hénaff et al., 2002 ; Costa et al., 2002). Cette méthode, quand sa fiabilité est prouvée, reste cependant imprécise (marge d'erreur probablement $\geq 10 \mathrm{~m}$ et très variable). C'est néanmoins la seule façon d'évaluer le recul des falaises sur une période longue (154 ans dans le cas qui nous intéresse). Les cadastres utilisés (1833, 1834, 1935 et
1987) ont fait l'objet d'une numérisation, puis d'une vectorisation pour faciliter la superposition des calques informatiques.

L'échelle suffisamment précise $(1 / 11000)$ des photographies aériennes de l'IGN (Institut Géographique National) prises le 20/04/1939 a permis d'établir par restitution photogrammétrique au $1 / 2000$, la position du haut de la falaise de Strouanne il y a 65 ans, avec une marge d'erreur de $\pm 1 \mathrm{~m}$. Les points de calage utilisés, au minimum quatre par cliché, ont été repérés sur les photographies de 1939 (calvaires, bâtiments, et intersections de routes) et mesurés par GPS sur le terrain. Les valeurs de recul de la falaise ont été obtenues par recalage, dans le système IGN Lambert 1, sur un plan topographique également établi par procédés photogrammétriques (marge d'erreur $\pm 14 \mathrm{~cm}$ ) à partir d'une mission aérienne effectuée le 28/03/2002 (échelle des clichés : $\left.1 / 7500^{\circ}\right)$. Le recul du haut de la falaise a ensuite été mesuré tous les cinq mètres, perpendiculairement à l'orientation générale du trait de côte $\left(\mathrm{N} 45^{\circ}\right)$. La même démarche a été appliquée pour la falaise de Sangatte, à partir de clichés du $20 / 09 / 1944$ au $1 / 7000^{\circ}$ de la Royal Air Force, permettant de couvrir une période un peu plus courte de 58 ans (la couverture de 1939 n'atteint pas le secteur). Cette méthode, contrairement à celle utilisant les cadastres, donne des résultats d'une grande précision (Overton et al., 1996 ; Lahousse et al., 2000 ; Moore, 2000 ; Catalao et al., 2000, 2002 ; Costa et al., 2001).

Le rythme de recul pour la période 1939 2003 a été déterminé à partir de l'analyse de

Interglaciaire intra-saalien Intra-Saalian interglacial stage

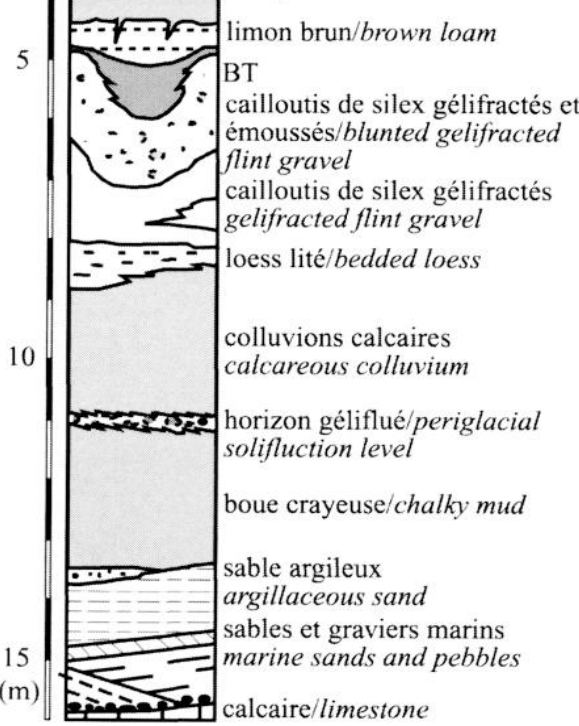

Holocène/Holocene
Weichselien/Weichselian
Eémien/Eemian
Saalien/Saalian
Interglaciaire intra-saalien
Intra-Saalian interglacial stage
Saalien/Saalian
Holsteinien/Holsteinian
Crétacé/Cretaceous

Fig. 3 - Lithostratigraphie des dépôts pléistocènes de Sangatte au puits du tunnel sous la manche (d'après Sommé et al., 1999, modifié).

Fig. 3 - Lithostratigraphy of the Pleistocene deposits in the Channel Tunnel pit (after Sommé et al., 1999, modified).

Fig. 4 - Lithostratigraphie de la falaise de Sangatte. 1 : dépôts de versant non calcaires ; 2 : dépôts de versant calcaires ; 3 : marnes ; 4 : éboulis crayeux ; 5 : sables marins ; 6 : galets marins ; 7 : craie turonienne; $8:$ craie cénomanienne (d'après Sommé et al., 1999, modifié).

Fig. 4 - Lithostratigraphy of Sangatte cliff. 1: non calcareous slope deposits; 2: calcareous slope deposits; 3: marls; 4: chalky screes; 5 : marine sands; 6 : marine pebbles; 7: Turonian Chalk; 8: Cenomanian Chalk (after Sommé et al., 1999, modified).

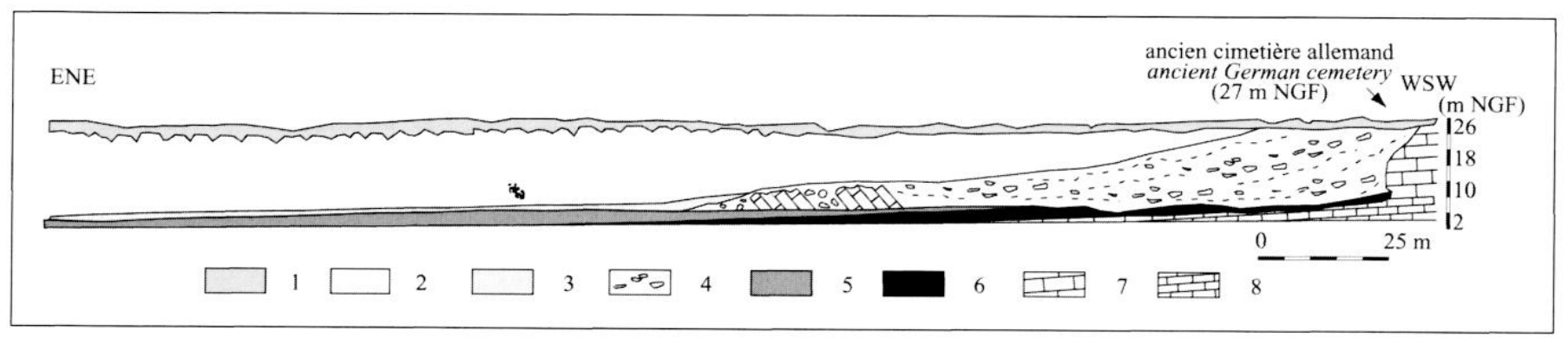




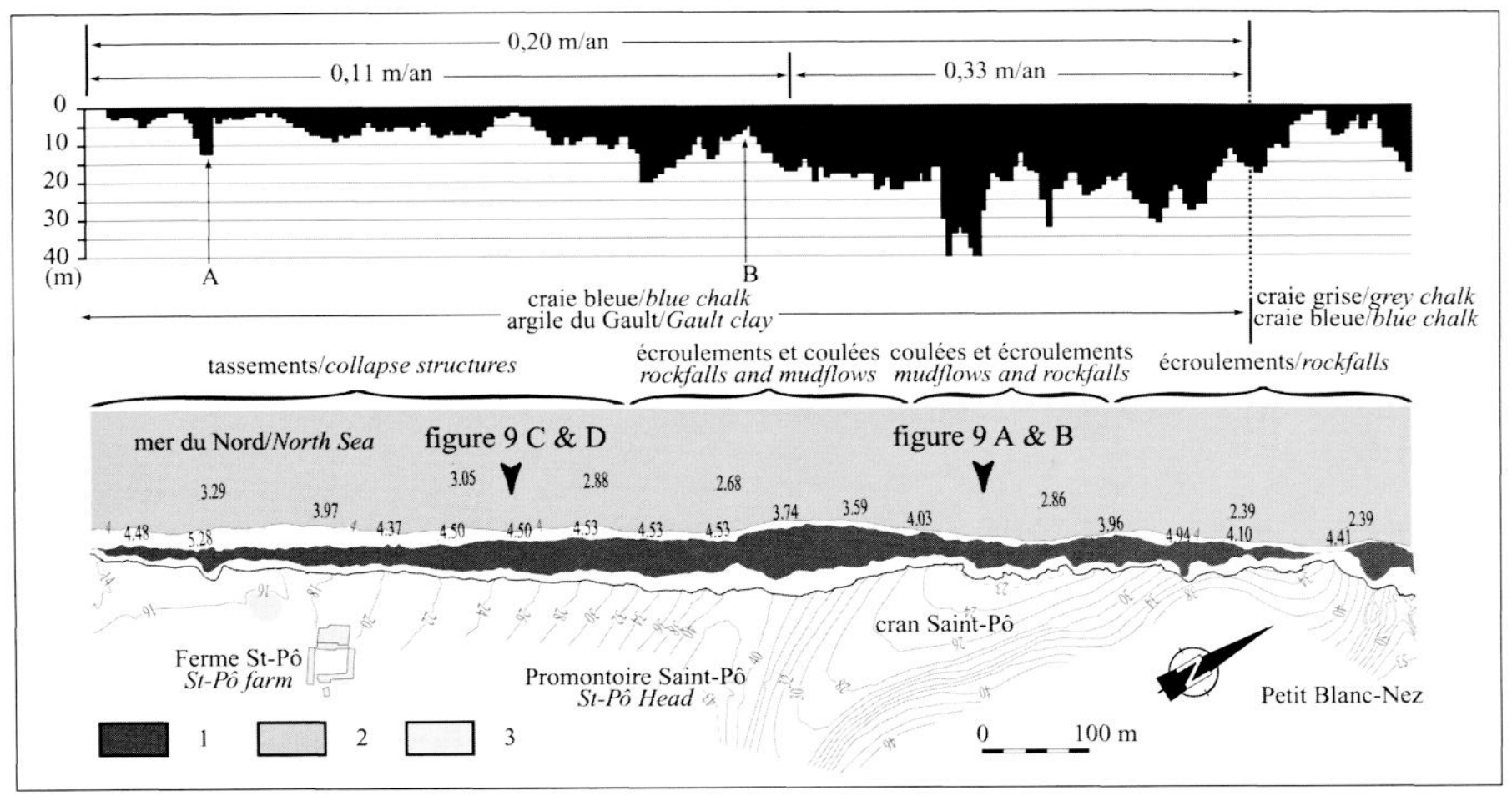

Fig. 5 - Valeurs et modalités de recul pour la falaise de Strouanne. 1 : matériaux déplacés (craie et argile ; la limite vers la mer correspond au bas de la falaise) $; 2$ : haute mer de coefficient $111 ; 3:$ mare artificielle.

Fig. 5 - Amount and mode of retreat at the Strouanne cliff. 1: displaced materials (chalk and clay; the boundary toward the sea is the bottom of the cliff); 2: high tide, coefficient 111; 3: artificial pond.

Fig. 6 - Valeurs et modalités de recul pour la falaise de Sangatte. $1:$ cordon de galets $; 2$ : haut et bas de falaise $; 3:$ haute mer de coefficient 111.

Fig. 6 - Amount and mode of retreat at the Sangatte cliff. 1: shingle bar; 2: top and bottom of the cliff; 3: high tide, coefficient 111.

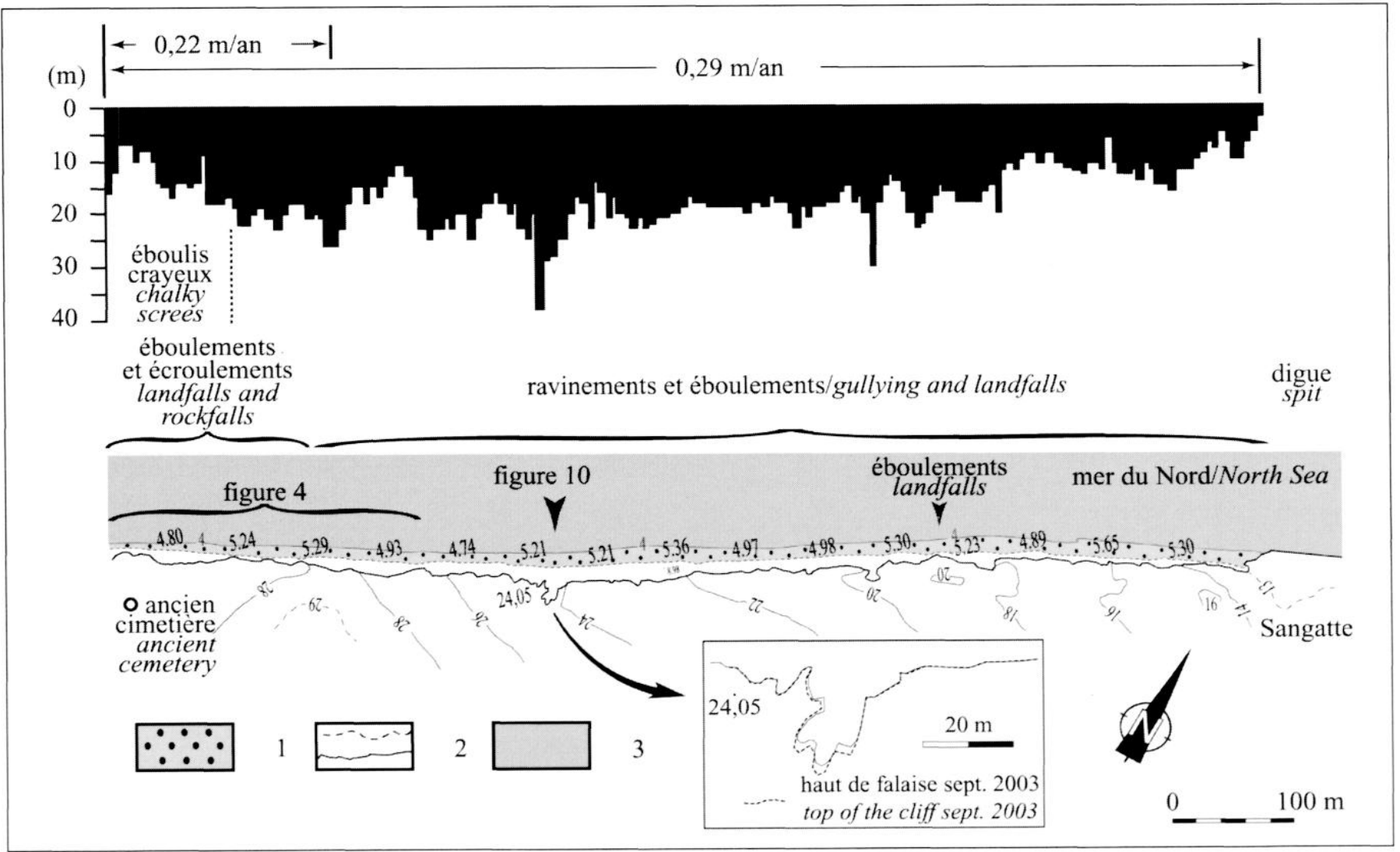


28 missions aériennes (vues verticales ; 24 missions IGN d'échelle comprise entre $1 / 11500$ et $1 / 300(0), 1$ mission RAF à $1 / 7000$ (), 3 missions spécifiques à 1/750(0)', des archives du journal la Voix du Nord (depuis 1960) et de la Direction Régionale de l'Environnement du Nord-Pas-deCalais (vues obliques), et enfin de panoramas pris depuis l'estran (Wissocq, 1990 - in 1992 ; nous-mêmes en 1999, $2000), 2001,2002$ et 20(03). Ces documents ont permis de dénombrer les écroulements et de suivre l'évolution des coulées. À deux ans d'intervalle, et après implantation de points de repère, quatre levés topographiques ont été effectués au tachéomètre à visée infrarouge (précision d'ordre millimétrique). Les données numériques ont ensuite été transférées vers un logiciel de traitement (SURFER v.7.()) permettant d'obtenir une traduction cartographique sous forme de Modèle Numérique de Terrain. Les fonds topographiques obtenus (équidistance des courbes de niveau : $0,2 \mathrm{~m}$ ) ont servi à l'établissement de croquis géomorphologiques interprétatifs illustrant les processus d'évolution de la falaise de Strouanne.

\section{Rythmes et valeurs de recul}

\section{Rythme de recul}

Les photographies au sol et aériennes obliques permettent d'identifier et de localiser les masses écroulées quelle que soit leur taille, et les vues aériennes verticales celles de taille supéricure à quelques centaines de mètres cubes. Sur la figure 7 sont localisées les plus volumineuses d'entre elles $(>1000 \mathrm{~m}$ ' environ), afin de mettre en valeur les périodes d'instabilité majeure. L'évolution des lobes et des pans glissés est consignée sur le tableau 1. Pour la falaise de Sangatte, un tel comptage est impossible : le pied de falaise est bordé d'un talus continu, alimenté en permanence par des éboulements de taille réduite.

L'analyse diachronique à partir des documents photographiques fait ressortir les éléments suivants : l'instabilité de ces falaises est un fait constant sur les 64 années considérées ; les mouvements de terrain ont un caractère ubiquiste ; les variations structurales interviennent plus dans la nature des mouvements que dans leur occurrence ; la fréquence de ces mouvements est élevée. Il ne se passe pas une année sans événement nouveau, même minime $(\leq 100) \mathrm{m}$ ' pour les masses écroulées); la hauteur de falaise influence la valeur du recul instantané. De ce fait, le recul au promontoire Saint Pô, où la falaise est aux trois quarts crayeuse, s'effectue à un rythme plus lent, compensé par des volumes plus importants, comme c'est le cas au Petit Blanc-Nez et au Blanc-Nez (Pierre et Lahousse, 2003).

\section{Valeurs de recul à Strouanne}

À Strouanne, la comparaison des cadastres de 1833 et de 1987 (commune de Wissant) permet de mesurer le recul à moyen terme (fig. 8). Cette comparaison met en évidence (tab. 2) : 1) l'hétérogénéité des valeurs de recul observées : de moins de $10 \mathrm{~m}$ (soit moins de $0,06 \mathrm{~m} / \mathrm{an}$ ) à près de $40 \mathrm{~m}$ (soit $0,26 \mathrm{~m} / \mathrm{an}$ ) ; 2) près d'un tiers du linéaire a reculé de plus de $30 \mathrm{~m}$ en 154 ans, soit un recul annuel moyen de $0,19 \mathrm{~m} / \mathrm{an} ; 3$ ) un recul globalement plus faible (inférieur à $20 \mathrm{~m}$, soit $0,13 \mathrm{~m} / \mathrm{an}$ ) là où la hauteur de la falaise n'excède pas $15 \mathrm{~m}$ (vers la ferme Saint-Pô). De telles valeurs sont cependant relativement rares puisqu'elles n'affectent que $15,3 \%$ de la totalité du linéaire concerné.

La méthode photogrammétrique, sur un pas de temps plus court (64 et 58 ans), permet d'affiner ces résultats en mettant

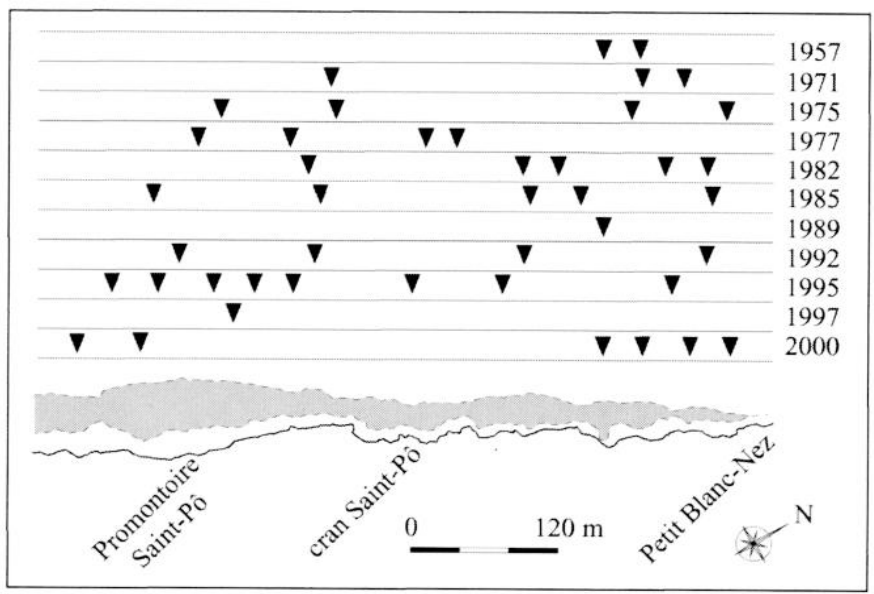

\begin{tabular}{|c|c|c|}
\hline $\begin{array}{c}\text { Lobes et pans glissés visibles } \\
\text { Lobes and planar slides }\end{array}$ & $\begin{array}{c}\text { Pans glissés visibles } \\
\text { Planar slides }\end{array}$ & $\begin{array}{c}\text { Pas d'événements apparents } \\
\text { No apparent events }\end{array}$ \\
\hline 1939 & 1971 & 1963 \\
1949 & 1975 & 1969 \\
1957 & 1977 & 1970 \\
1981 & 1992 & 1976 \\
1982 & & 1979 \\
1983 & & 1988 \\
1985 & & 1989 \\
1986 & & 1990 \\
1995 & & 1997 \\
nov. 2000 & & 1999 \\
2002 & & juin 2000 \\
2003 & & 2001 \\
\hline
\end{tabular}

Fig. 7 - Localisation des écroulements repérés sur photographies, entre le promontoire Saint-Pô et le Petit Blanc-Nez.

Fig. 7 - Location of the rockfalls identified on photographs, between Saint-Pô Head and Petit Blanc-Nez.

Tableau 1 - Occurrence et types de mouvements de terrain liés à I'affleuremement de I'argile du Gault.

Table 1 - Occurrence and types of mass movement along the Gault Clay outcrop. 


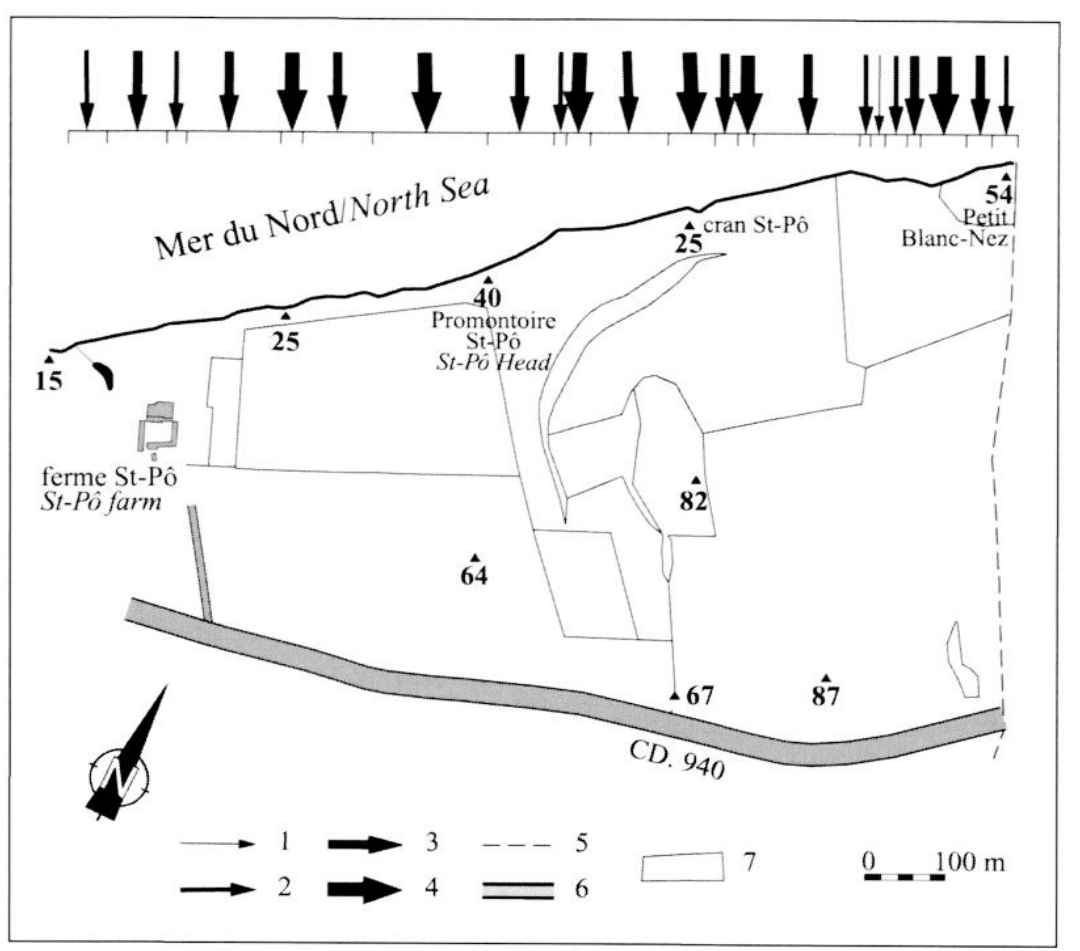

Fig. 8 - Valeurs de recul d'après le cadastre (18331987) pour la falaise de Sangatte (commune de Wissant). $1:<10 \mathrm{~m} ; 2:$ de 10 à $20 \mathrm{~m} ; 3:$ de 20 à $30 \mathrm{~m} ; 4$ : de 30 à $40 \mathrm{~m} ; 5$ : limite communale ; 6 : voirie $; 7$ : parcelle.

Fig. 8 - Amount of retreat inferred from cadastral registers (1833-1987), Sangatte cliff (municipality of Wissant). 1: $10 \mathrm{~m} ; 2: 10$ to $20 \mathrm{~m} ; 3: 20$ to $30 \mathrm{~m}$; 4: 30 to $40 \mathrm{~m}$; 5 : municipal boundary; 6 : roads; 7 : plot.

Tableau 2 - Valeurs de recul établies par la méthode cadastrale entre le Petit Blanc-Nez et Strouanne (1833-1987).

Table 2 - Amount of retreat between Petit BlancNez and Strouanne after comparison from cadastral registers (1833-1987).

en avant la variabilité spatiale des valeurs de recul. Celle-ci est largement conditionnée par la hauteur de la falaise et par la topographie du revers (fig. 5). Un premier secteur s'individualise entre Strouanne et le promontoire Saint-Pô, avec un recul annuel moyen de $0,11 \mathrm{~m} / \mathrm{an}$. Les valeurs de recul brut, comprises entre 0 et $20 \mathrm{~m}$, s'accroissent avec la hauteur de la falaise, mais avec une irrégularité traduisant le caractère aléatoire des mouvements. Celle-ci

\begin{tabular}{|c|c|c|}
\hline $\begin{array}{c}\text { Recul brut (en m) } \\
\text { Total retreat }(\boldsymbol{m})\end{array}$ & $\begin{array}{c}\text { Recul annuel moyen (en m/an) } \\
\text { Mean annual retreat }(\boldsymbol{m} / \mathbf{y r})\end{array}$ & $\begin{array}{c}\% \text { du linéaire } \\
\text { Coastline } \%\end{array}$ \\
\hline$<10$ & $<0,06$ & 1,4 \\
$10-20$ & $0,06-0,13$ & 13,9 \\
$20-30$ & $0,13-0,19$ & 56,3 \\
$30-40$ & $0,19-0,26$ & 28,4 \\
\hline
\end{tabular}
est d'autant mieux marquée au promontoire que les valeurs maximales de recul y sont les plus fortes. Dans cet ensemble, deux segments s'individualisent. Le premier, au sud-ouest de la mare artificielle de la ferme Saint-Pô, a reculé d'une douzaine de mètres depuis 1939 , pour une hauteur de falaise d'à peine $10 \mathrm{~m}$ (en A fig. 5). Cette anomalie s'explique par l'épaisseur de craie bleue qui est minimale à cet endroit, mais aussi par la présence de la mare qui entraîne une multiplication des sources au contact de l'argile du Gault. La faible valeur de recul, $5 \mathrm{~m}$ en 64 ans, relevée au sommet du promontoire Saint-Pô $(40 \mathrm{~m})$, correspond à une section située à la limite entre deux niches d'arrachement voisines (en B fig. 5). Le second secteur, axé sur le cran Saint-Pô, recule plus vite $(0,33 \mathrm{~m} / \mathrm{an})$ et plus régulièrement, bien que la falaise s'y abaisse jusqu'à $23 \mathrm{~m}$. La valeur la plus forte $(40 \mathrm{~m}$, soit $0,63 \mathrm{~m} / \mathrm{an})$, se trouve logiquement à l'exutoire du cran Saint-Pô, dont la position se décale vers l'ouest au fur et à mesure du recul du haut de falaise qui recoupe obliquement le vallon (fig. 5).

\section{Valeurs de recul à Sangatte}

À Sangatte, les trois cadastres disponibles (1834, 1935 et 1987) apparaissent moins fiables, puisqu'ils suggèrent une augmentation des valeurs de recul vers Sangatte pour les deux périodes (1834-1987 et 1935-1987). et une quasi-

absence de recul (entre 1932 et 1987) pour une section située à environ $150 \mathrm{~m}$ au nord-est du cimetière allemand ; autant de données démenties par l'analyse photogrammétrique faite à partir des clichés de 1944 et 2002, ainsi que par l'étude des photographies depuis 1987. Ces données ne sont donc pas exploitables. L'analyse par photogrammétrie permet à nouveau de diviser en deux secteurs distincts le linéaire étudié (fig. 6). Le premier secteur se situe entre l'ancien cimetière et une petite élévation $(29 \mathrm{~m})$ venant interrompre la pente générale du versant reliant le Blanc-Nez à Sangatte. Le recul moyen annuel y est de $0,22 \mathrm{~m} / \mathrm{an}$ et les valeurs augmentent graduellement d'ouest en est. Le second secteur $(0,29 \mathrm{~m} / \mathrm{an})$ s'étire jusqu'à la digue de Sangatte. Il est caractérisé par une homogénéité du recul, abstraction faite de deux valeurs extrêmes à l'emplacement de deux profondes ravines. La diminution rapide des valeurs de recul vers l'est s'explique probablement par la présence de la digue de Sangatte, point fixe sur lequel la falaise est ancrée.

\section{Modalités du recul}

\section{Falaise de Strouanne : coulées et tassements}

Le suivi photographique depuis 1999 et la comparaison de deux cartes topogéomorphologiques levées en mai 2001 et mars 2003 au débouché du cran Saint-Pô (fig. 9 A et B) per- 
met de suivre la dynamique des coulées boueuses qui affectent ici la falaise. En juin 1999, l'argile du Gault est taillée en falaise sur deux mètres de haut par l'érosion marine, ce qui suggère un répit de l'action des processus continentaux. La surface de l'affleurement du Gault est alors régulière et en pente faible. En décembre 2000, en pleine activité
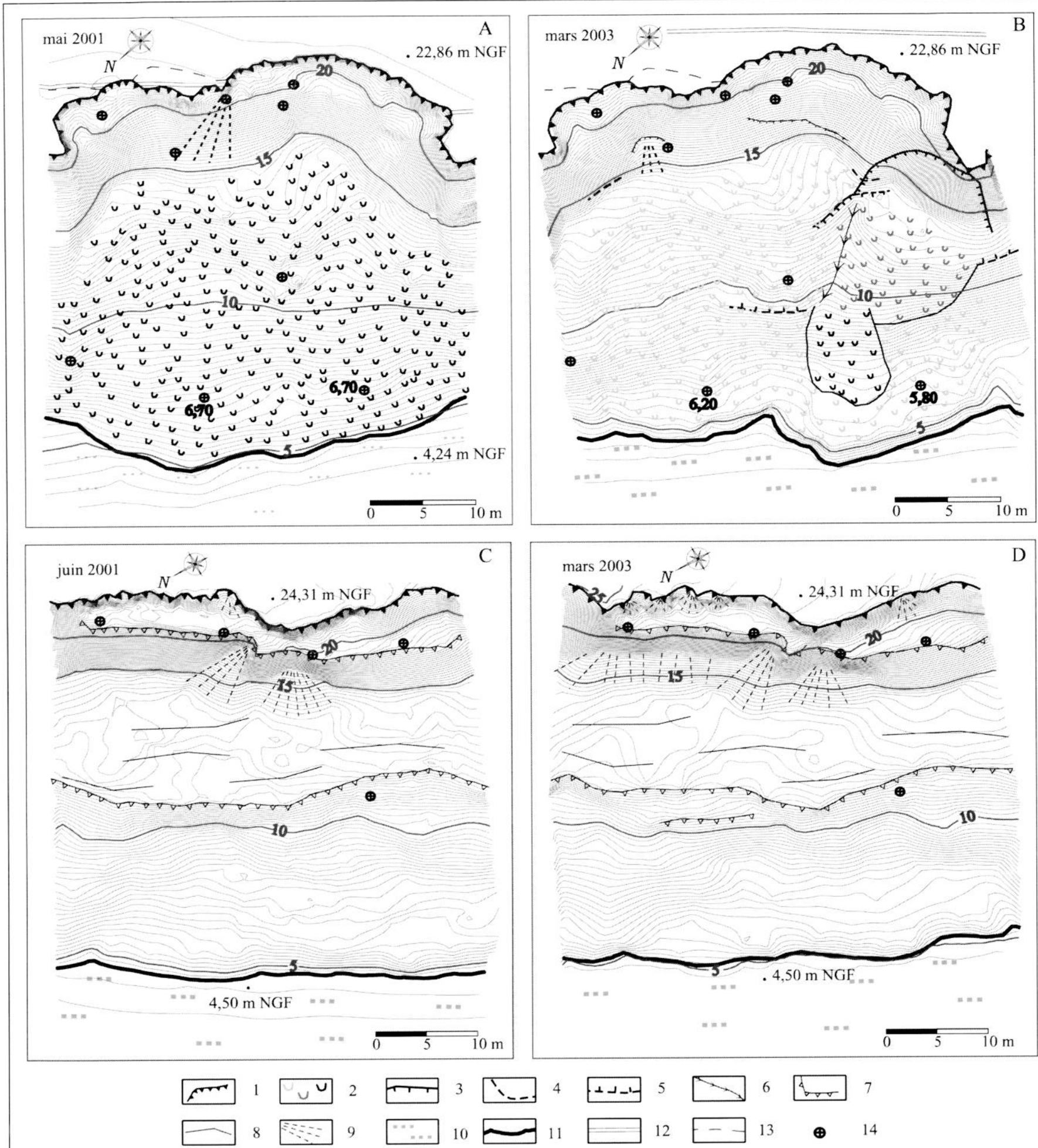

Fig. 9 - Carte géomorphologique des mouvements de terrain. A et $B:$ cran Saint-Pô, $C$ et $D:$ ferme Saint-Pô. $1:$ haut de falaise $; 2:$ coulées successives ; 3 : niche d'arrachement ; 4 : toit de l'argile du Gault in situ ; $5:$ argile du Gault déplacée ; $6:$ ravinement ; $7:$ pan glissé ; 8 : fissure ; 9 : éboulis ; 10 : estran ; 11 : front de coulée ; 12 : sentier ; $13:$ piquets. Équidistance des courbes de niveau :20 cm.

Fig. 9 - Geomorphological map. A and B: the cran Saint-Pô, $C$ and D: Saint-Pô farm. 1: top of the cliff; 2: successive mass movements; 3: scar; 4: roof of the Gault Clay, in situ; 5: Gault Clay, displaced; 6: gullying; 7: slipped mass; 8: crack; 9: scree; 10: strand zone; 11: toe of the flow; 12: trail; 13: landmarks. Contour interval: $20 \mathrm{~cm}$. 
(Lahousse et Pierre. 20(03a). la coulée s'allonge et sa pente s'accentue. entraînant vers le bas des volumes considérables de craie bleue déstructurée et de formations superficielles limoneuses. Le recul du haut de la falaise atteint alors 5 mètres (fig. 9 A), recoupant le sentier du littoral, et une fissure s'ouvre sur le revers. Le front de la coulée, d'où s'échappe le trop plein d'eau, décrit un large lobe qui se raccorde en pente douce à l'estran. La carte géomorphologique de mai 2001 (fig. 9 A et $\mathrm{B}$ ) illustre le résultat de cette dynamique et signale la position de neuf des vingt-deux piquets de fer implantés avant le levé de la carte topographique, encore visibles en mars 2003.

Deux ans plus tard, le haut de falaise et du versant n'ont quasiment pas bougé, comme l'indique la position des piquets implantés au printemps 2001 , mais la coulée a pris un profil concave (l'aplatissement de sa partie aval est compris entre 50 et $90 \mathrm{~cm}$ ) et le lobe s'est évasé de quelques mètres. Le front de la coulée a été sapé par la mer, car le contact avec l'estran est maintenant marqué par une microfalaise. Les processus continentaux marquent donc le pas, mais le site n'est pas pour autant stabilisé. Durant l'hiver 2003 , sous l'effet du fluage de l'argile du Gault, une partie des matériaux ameublis (formations superficielles et craie bleue altérée) qui affleurent sur les pentes fortes de la niche d'arrachement se détache et forme une coulée qui atteint la cote $8,40 \mathrm{~m}$. Un deuxième arrachement se produit ensuite dans l'argile du Gault (vers $13 \mathrm{~m}$ ), entraînant la formation d'une nouvelle coulée qui remanie en partie la précédente et atteint la cote $6 \mathrm{~m}$. À l'aplomb de ces mouvements, le front de la coulée initiale, qui avait été régularisée par la mer, avance à nouveau de quelques mètres, tout en conservant une pente forte (fig. $9 \mathrm{~A}$ et $\mathrm{B}$ ).

Cette succession d'événements, dans un contexte de forte pluviométrie d'abord (tab. 3 et tab. 4), puis d'un retour à la normale à partir de l'automne 2001, permet de rendre compte de la nature des mouvements décrits. En effet, le volumineux arrachement et la coulée de l'hiver 2000-2001, bien qu'exacerbés par le fluage de l'argile du Gault, s'expliquent largement par la très forte teneur en eau de la craie bleue altérée et des formations superficielles limoneuses qui la recouvrent, lesquelles s'écoulent sans qu'il y ait de plan de cisaillement bien défini. La concentration des eaux de ruissellement dans le cran Saint-Pô explique donc non seulement les valeurs élevées de recul à cet endroit, mais aussi les modalités de ce recul. Dans des conditions moins pluvieuses, l'argile du Gault, là où elle est exposée aux intempéries (c'est-à-dire sur la paroi), flue et déclenche en fonction de la cohérence de la craie des écroulements ou des coulées plus modestes. En arrière de la falaise, protégée par la craie bleue imperméable. l'argile ne s'humecte pas et reste stable.

Au sud du promontoire Saint-Pô, la falaise s'abaisse à nouveau et l'épaisseur de craie diminue à l'affleurement. En outre, les deux couches argileuse et crayeuse n'ont plus le même comportement : la craie, plus cohérente, se détache par pans et se tasse sous l'effet du fluage de l'argile dont le mouvement est plus lent. Cette portion du trait de côte ne présente que rarement des lobes de coulée sur les photographies aériennes. La situation de juin 2001 (fig. $9 \mathrm{C}$ et D) montre deux pans de craie (à $20 \mathrm{~m}$ et $14 \mathrm{~m}$ ), à structure conservée, sauf pour le plus bas des deux, tandis que sous $10 \mathrm{~m}$, le mouvement prend l'allure d'une coulée à blocs de craie. La craie déstructurée et l'argile présentent alors une topographie chaotique.

En mars 2003 le haut de falaise n'a quasiment pas bougé, comme l'indique la position des piquets implantés au printemps 2001, pas plus que la topographie du versant (fig. $9 \mathrm{C}$ et D). La coulée a essentiellement évolué par recul du front, ce qui indique que depuis deux ans, le sapement par la mer est plus actif que le fluage de l'argile du Gault. Le palier intermédiaire s'est fragmenté en un point, mais quelques mètres plus au sud un piquet conserve sa position en $X, Y$ et $Z(11,20 \mathrm{~m})$. Le palier supérieur, globalement stable, se tasse d'une vingtaine de centimètres à ses deux extrémités et se segmente en s'éboulant dans sa partie médiane. Les éboulis crayeux sur les pentes fortes, produits par la gélifraction et la dessiccation, sont mieux conservés.

Tableau 3 - Précipitations mensuelles en $\mathrm{mm}$ et moyennes mensuelles (1970-1999) à Guines (source : Eaux de Calais).

Table 3 - Monthly rainfalls in $\mathrm{mm}$ and average monthly rainfall (1970-1999) (Guines, after Eaux de Calais).

\begin{tabular}{|c|c|c|c|c|c|c|c|c|c|c|c|c|c|}
\hline & Janv. & Fév. & Mars & Avril & Mai & Juin & Juil. & Août & Sept. & Oct. & Nov. & Déc. & Année \\
\hline 1999 & 112,4 & 46,8 & 63,2 & 62,8 & 59,2 & 77,7 & 5,1 & 128,5 & 134,5 & 77,2 & 53 & 197,6 & 1018 \\
\hline 2000 & 53,2 & 69,5 & 37,1 & 122,4 & 87,6 & 26,5 & 63,8 & 39,3 & 84 & 212,4 & 237,6 & 111,7 & 1145,1 \\
\hline 2001 & 123,4 & 80,3 & 126,7 & 110,1 & 34 & 20,4 & 62,6 & 75,4 & 175,6 & 132,2 & 108,2 & 67,8 & 1116,7 \\
\hline 2002 & 63 & 126,8 & 58 & 38,6 & 84 & 88 & 70,2 & 30,2 & 55,2 & 62,2 & 123,4 & 106,4 & 906 \\
\hline 2003 & 81 & 25,8 & 41,8 & 49 & 53 & 52,8 & 47,8 & 60,8 & 27,6 & 64,6 & 44,4 & 64,6 & 613,2 \\
\hline Moy. & 77,8 & 50,2 & 58,9 & 52,2 & 52,7 & 57,2 & 49,2 & 57 & 84,7 & 94,1 & 98,4 & 89,7 & 822 \\
\hline
\end{tabular}

\begin{tabular}{|l|l|c|c|c|c|c|c|c|c|c|c|c|c|c|c|c|c|c|c|c|c|c|c|c|c|c|c|c|c|c|}
\hline $\mathbf{2 9}$ & $\mathbf{3 0}$ & $\mathbf{3 1}$ & $\mathbf{1}$ & $\mathbf{2}$ & $\mathbf{3}$ & $\mathbf{4}$ & $\mathbf{5}$ & $\mathbf{6}$ & $\mathbf{7}$ & $\mathbf{8}$ & $\mathbf{9}$ & $\mathbf{1 0}$ & $\mathbf{1 1}$ & $\mathbf{1 2}$ & $\mathbf{1 3}$ & $\mathbf{1 4}$ & $\mathbf{1 5}$ & $\mathbf{1 6}$ & $\mathbf{1 7}$ & $\mathbf{1 8}$ & $\mathbf{1 9}$ & $\mathbf{2 0}$ & $\mathbf{2 1}$ & $\mathbf{2 2}$ & $\mathbf{2 3}$ & $\mathbf{2 4}$ & $\mathbf{2 5}$ & $\mathbf{2 6}$ & $\mathbf{2 7}$ & $\mathbf{2 8}$ \\
\hline 10 & 28 & 35 & 16 & 27 & 10 & 3,8 & 22 & 7,4 & 13 & 0 & 0 & 4,4 & $\mathbf{9}$ & $\mathbf{8}$ & $\mathbf{8}$ & 5,5 & 5,9 & 3,6 & 0,3 & 5,3 & 4,8 & 40 & 9,5 & 6,4 & 7 & 0,3 & 13 & 1,5 & 5 & 0,1 \\
\hline
\end{tabular}

Tableau 4 - Précipitations journalières en mm (29 octobre28 novembre 2000) (Guines, source : Eaux de Calais).
Table 4 - Daily rainfalls in $\mathrm{mm}$ (October 29-November 28, 2000) (Guines, after Eaux de Calais). 


\section{Falaise de Sangatte : ravinements et éboulements}

La lithostratigraphie des dépôts pléistocènes de Sangatte, telle qu'elle a été levée dans le puits du tunnel sous la Manche (Sommé et al., 1999) est synthétisée sur la figure 3. Cette succession se retrouve sans grand changement le long de la falaise, sauf à l'aplomb de l'ancien puits, où une masse d'éboulis crayeux s'intercale entre les sables et gravicrs marins et les formations litées périglaciaires (fig. 4).

Les formations litées de la falaise de Sangatte reculent sous l'effet conjugué de l'ouverture de fissures de détente, de l'infiltration des eaux de pluie et du ruissellement, comme l'illustre la figure 10. Les eaux du revers de la falaise s'infiltrent à la faveur de fissures de détente parallèles au rebord, et circulent dans les différents lits de la formation ( 1 et 2 sur la figure 10), plus ou moins rapidement en fonction de leur granulométrie. Sur la paroi de la falaise, l'eau exfiltrée par les niveaux les plus perméables entraîne avec elle des éléments fins. Les alvéoles de soutirage (3) qui en résultent guident le ruissellement, et le ravinement gagne le haut de la paroi, puis entaille le revers par érosion régressive et s'ap-

Fig. 10 - Modalités de recul de la falaise de Sangatte. 1 : formations litées (limons, boue, cailloutis à silex) ; 2 : formations caillouteuses et sableuses ; 3 : exfiltration et soutirage ; 4 : cônes de déjection ; 5 : éboulis.

Fig. 10 - Mode of retreat at the Sangatte cliff. 1: bedded deposits (loam, mud, gravel with flint); 2: gravelly and sandy deposits; 3: point of emergence and suffosion; 4: alluvial cone; 5: scree.
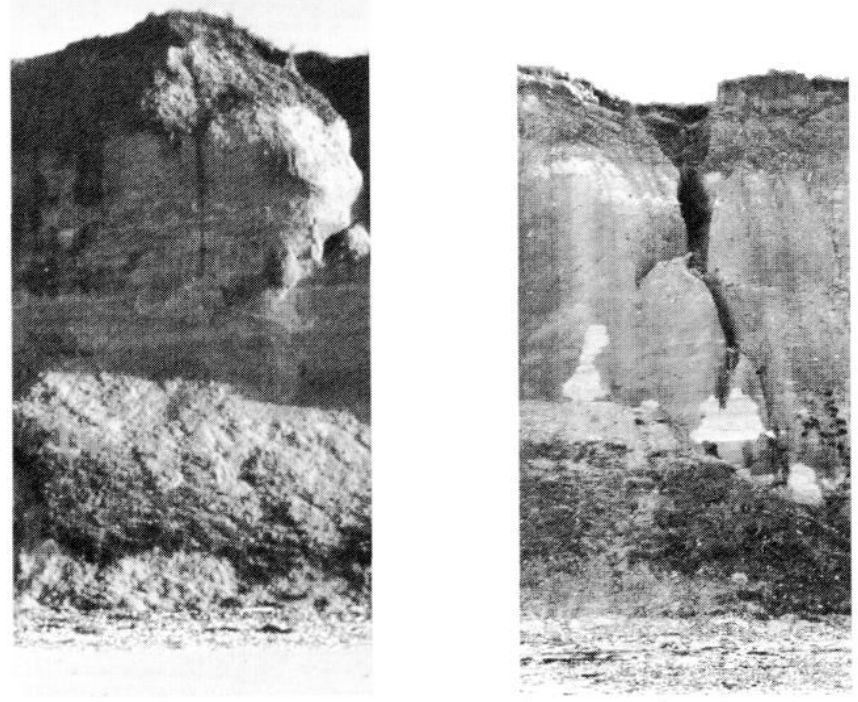

éboulement liquide liquid landfall mars 2003 desquamation desquamation janvier 2002
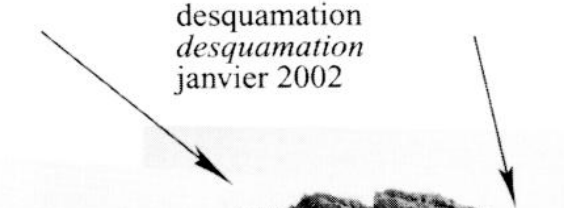

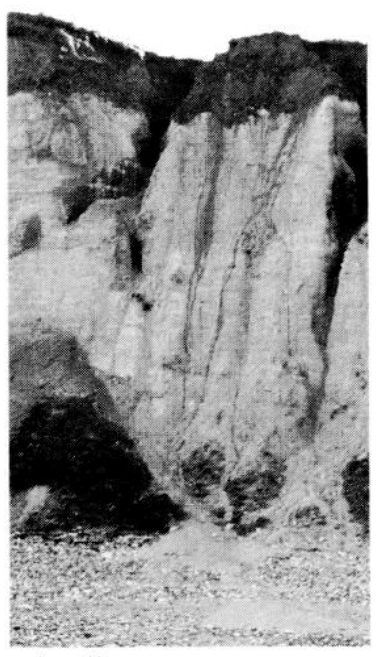

\section{ruissellement}

runoff

janvier 2003

\section{4,05 m NGF}

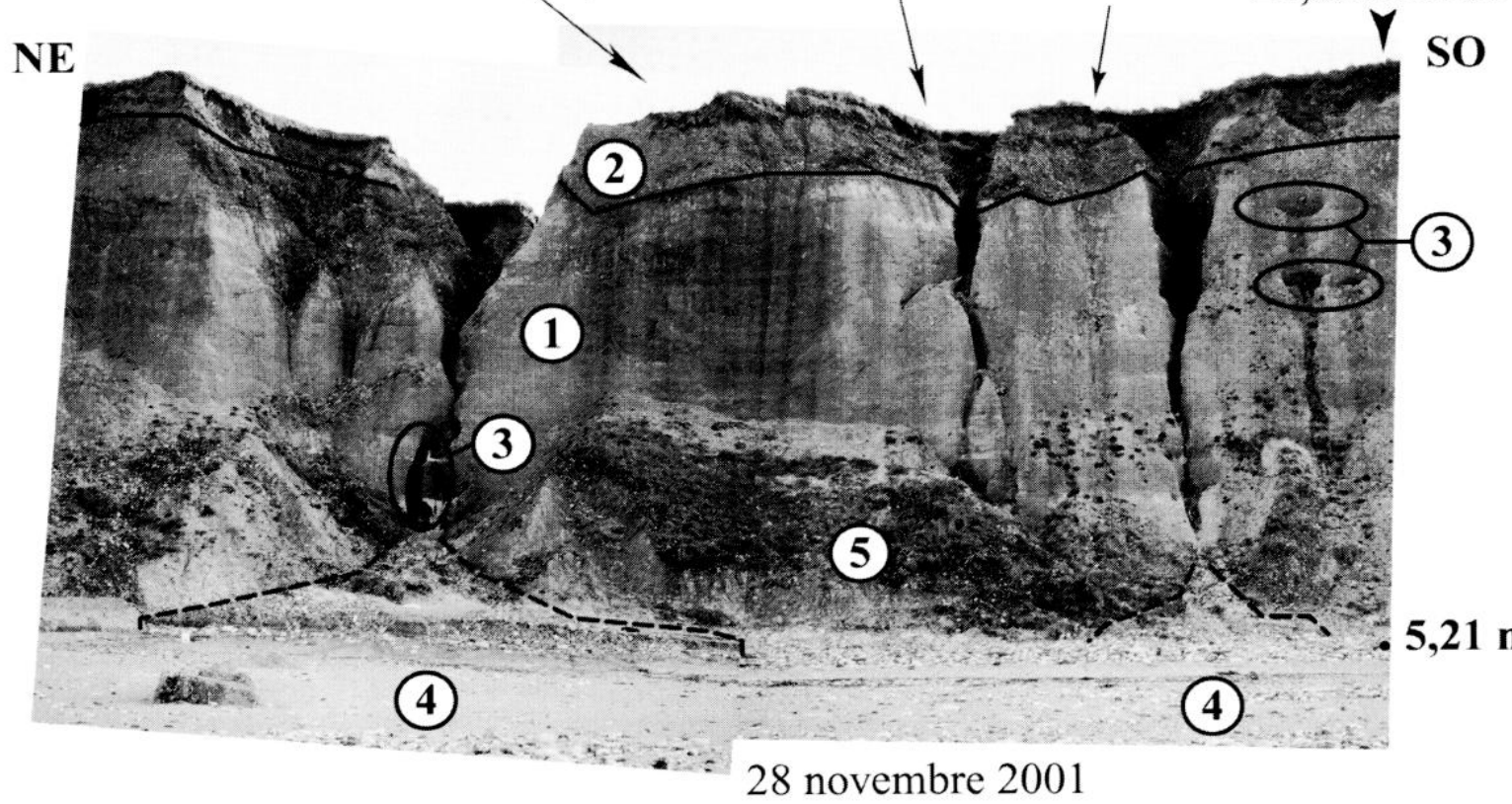


profondit. Au débouché des ravines évoluées se forment des cônes de déjection (4) (sapés par la mer sur la photographie) qui entaillent le cordon de galets et les éboulis accumulés en pied de falaise. Le ravinement, qui peut prendre une forme digitée, isole éventuellement des pans limoneux dont la densité a diminué du fait du soutirage : ils se tassent (suffosion) puis s'éboulent (5), ou éventuellement basculent.

Entre 2001 et 2003, la falaise de Sangatte n'est pas moins figée que celle de Strouanne, mais les processus qui l'affectent de façon continue sont plus discrets. Deux petits éboulements affectent le haut de la falaise vers Sangatte, tandis que la ravine décrite en novembre 2001 et cartographiée en mars 2002 gagne sur les champs par érosion régressive (fig. 6). Deux processus affectant la paroi ont encore été observés : éboulement de matériaux liquides et desquamation (fig. 10). Au total, la quantité de débris meubles accumulés au pied de la falaise tient à la fois au fait que la mer n'atteint des cotes supérieures à $4 \mathrm{~m}$ que par temps houleux et que la recharge en matériaux est quasi-continue. Leur évacuation se fait lentement et principalement sous l'action du ruissellement des eaux continentales. Ces processus n'agissent plus avec la même efficacité là où les éboulis crayeux pléistocènes affleurent, ce qui explique les modalités de recul hybrides à l'aplomb de l'ancien puits : éboulements dans les formations limoneuses et petits écroulements dans les éboulis calcaires.

\section{Discussion}

La cartographie précise des valeurs et des modalités de recul met en avant une variabilité spatiale dont l'analyse permet de déterminer le rôle respectif des différents paramètres, structuraux, topographiques et hydrologiques, qui influencent l'évolution des falaises de Strouanne et de Sangatte, secteur par secteur.

Les mouvements de terrain qui affectent la falaise de Strouanne sont déterminés à la fois par la géométrie de l'affleurement (rapport argile/craie ; fig. 2), par la hauteur de la paroi et par la topographic du revers de la falaise. Les deux premiers paramètres combinés conditionnent en outre la longueur et la valeur des segments de pente dans la craie et dans l'argile, donc le profil de la falaise. Ainsi, en partant du Petit Blanc-Nez, tant que l'argile du Gault n'affleure que sur 2 à 3 mètres maximum et pour des hauteurs de paroi supérieures à $25 \mathrm{~m}$, les écroulements dominent. Mais les valeurs et le rythme du recul changent radicalement en fonction de l'absence (au Petit Blanc-Nez) ou de la présence (vers l'ouest) de l'argile en pied de falaise : si l'argile affleure, la paroi est plus uniformément affectée sur 64 ans (fig. 5).

À partir du cran Saint-Pô, où l'argile albienne affleure presque intégralement (soit sur $11 \mathrm{~m}$ ), trois combinaisons de mouvement se succèdent en direction de Strouanne. 1) Au débouché du cran (altitude $23 \mathrm{~m}$ ), où l'argile s'étire sur une vingtaine de mètres en avant de la craie bleue altérée et des formations superficielles qui la recouvrent, les coulées l'emportent sur les écroulements. La présence même du cran explique les valeurs maximales du recul dans son axe : les eaux de surface drainées vers la mer modifient régulière- ment les caractéristiques géotechniques de l'argile du pied de la falaise, mais aussi celles de la craie bleue argilisée et des formations superficielles qui la recouvrent. 2) Au promontoire Saint-Pô $(40 \mathrm{~m})$, la craie bleue, en retrait d'une quarantaine de mètres du pied de la falaise, affleure dans son intégralité $(30 \mathrm{~m})$ et livre des écroulements volumineux mais peu fréquents, qui s'accumulent momentanément sur l'argile qui les évacue. 3) Vers la ferme Saint-Pô, enfin, à des altitudes inférieures à $30 \mathrm{~m}$, le rapport argile/craie bleue commence à s'inverser et les écroulements disparaissent complètement, laissant la place à des tassements de pans de craie.

L'hypothèse de Bonte (1959) selon laquelle ces falaises s'effondrent sous leur propre poids n'explique donc ici ni les valeurs, ni le rythme de recul. Alors que, selon cet auteur, l'argile humide ne devrait pas être chargée à plus de 3 à $5 \mathrm{~kg} / \mathrm{cm}^{2}$ pour rester stable, cette charge atteint $8,4 \mathrm{~kg} / \mathrm{cm}^{2}$ au promontoire Saint-Pô pour une hauteur de falaise de $40 \mathrm{~m}$ et $4,9 \mathrm{~kg} / \mathrm{m}^{2}$ au cran Saint-Pô pour une hauteur de $23 \mathrm{~m}$. Les valeurs maximales de recul depuis $1939(15 \mathrm{~m}$ et $40 \mathrm{~m}$ respectivement) y sont pourtant inversement proportionnelles aux charges (fig. 5), et la falaise dans son ensemble n'a que très peu évolué depuis 3 ans (hiver 2001-automne 2003), si ce n'est justement au cran Saint-Pô. La relation inversement proportionnelle entre la hauteur de la falaise et son recul (Guilcher, 1966 ; Costa et al., 2003), relation qui s'atténue à long terme (Sunamura, 1992), doit par ailleurs être nuancée à cette échelle d'analyse et pour ces matériaux. Entre le hameau de Strouanne et le promontoire Saint-Pô, la valeur du recul augmente avec la hauteur de la falaise, et au cran Saint-Pô le fort recul tient plus à la topographie sommitale de la falaise qu'à sa hauteur.

La falaise de Sangatte n'échappe pas au double déterminisme topographique et structural (fig. 1 et fig. 6). La forme particulière de ravinement décrite plus haut n'affecte la falaise qu'à l'est de la cote 28 m (extrémité du Mont Roty) où les eaux, drainées par le Fond Pignon, s'infiltrent sur les pentes douces de l'affleurement du Pléistocène. L'ancien cimetière allemand se trouve au contraire loin en aval du Fond du Guet, et les eaux de ruissellement y parviennent en moins grande quantité. La combinaison des processus d'infiltration, de soutirage et de ravinement n'opère donc plus. En outre, les éboulis crayeux adossés à la falaise fossile (fig. 4) ont un comportement mécanique qui s'apparente plus à celui de la craie, ce qui favorise des écroulements plus volumineux et de granulométrie plus grossière. Le rythme du recul y est de fait un peu ralenti et la vitesse de recul sur 59 ans un peu moindre que pour le reste de l'affleurement du Pléistocène, qui évolue cependant de façon assez homogène. Le rythme de recul sur l'ensemble du site du Blanc-Nez (entre Strouanne et Sangatte, fig. 1) apparaît donc très contrasté. À Strouanne, en effet, les sections à recul fort évoluent à un rythme rapide et les sections à recul modéré à un rythme lent, tandis qu'entre le Petit-Blanc-Nez et le Fond du Guet la falaise crayeuse évolue au contraire à un rythme inversement proportionnel aux valeurs de recul (Lahousse et Pierre. 2002). Il n'est donc pas possible de généraliser la relation entre valeur de recul et fréquence et amplitude des 
mouvements, même pour des lithologies comparables (Davies et al., 1998 ; Costa et al., 20)3).

Bien qu'il convienne de rester prudent quant à l'utilisation des cadastres, le trait de côte n'étant pas daté et son tracé probablement imprécis sur le cadastre de 1987 (Pierre et Lahousse, 20(03), la comparaison des résultats des études cadastrales et photogrammétriques fournit un indice supplémentaire sur le rythme d'évolution de la falaise de Strouanne. Pour une période de 154 ans prenant fin en 1987, le cadastre indique que les valeurs de recul sont maximales au cran Saint Pô (jusqu'à $40 \mathrm{~m}$ ), ainsi que juste à l'ouest de promontoire Saint-Pô et du Petit Blanc-Nez (jusqu'à 40 m également, fig. 8). Pour ces deux derniers endroits, les valeurs de recul sont bien supéricures à celles calculées sur 58 ans (comprises entre 6 et $20 \mathrm{~m}$ ), tandis qu'au cran Saint-Pô la valcur de recul est la même sur 154 ans et sur 58 ans. Ce constat peut s'expliquer de la façon suivante : le recul a dû s'accélérer depuis 1987 au cran où il est largement influencé par les précipitations. À ce titre, les précipitations de l'année 2000 et des premiers mois de l'année 2001 ont sans doute largement contribué à son recul récent (tab. 3). Au promontoire Saint-Pô et au Petit Blanc-Nez, en revanche, le rythme de recul plus aléatoire propre aux secteurs évoluant par écroulements trouve encore une illustration.

\section{Conclusion}

L'étude à grande échelle des processus, des rythmes et des valeurs de recul des falaises argilo-crayeuses et limoneuses qui prolongent vers le sud-ouest et vers le nord-est les falaises crayeuses du Blanc-Nez permet de préciser et de nuancer le rôle des paramètres structuraux, topographiques et hydrologiques dans l'évolution du site. La mesure précise du recul fournit en outre des données originales pour ce type de falaise.

À Sangatte et Strouanne, contrairement à ce qui se produit dans le secteur exclusivement crayeux, la mer n'a d'action érosive directe - en l'occurrence onde de choc des vagues et bombardement (Robinson, 1977 ; Sunamura, 1982 ; Kirkgöz, 1995 ; Benumof et al., 20(00)- que s'il y a conjonction d'un fort coefficient de marée et d'une tempête. À Strouanne, ces actions marines sporadiques induisent une régularisation du bas de falaise. Les processus continentaux prennent par contre une importance majeure dans la déstabilisation des deux falaises, comme c'est souvent le cas pour celles en roches meubles (Steers, 1962). La topographie de l'arrière de la falaise et la perméabilité des matériaux contrôlent l'infiltration et le ruissellement, ainsi que l'altération là où les pentes sont faibles (fond du cran Saint-Pô), tandis que le pendage des sédiments contrôle les exsurgences sur la paroi de la falaise (falaise de Sangatte). Topographie et structure expliquent donc largement les modalités et les valeurs de recul, le rythme de recul étant en outre influencé par le climat. À ce titre, il est légitime de se poser la question d'une accélération récente du rythme de recul, hypothèse que l'étude conjointe, sur un demi-siècle, du rythme d'évolution et des données climatologiques et hydrogéologiques permet d'aborder.

\section{Remerciements}

Ce travail a été réalisé avec le soutien financier de la Direction Régionale de l'Environnement du Nord Pas-deCalais. Ont collaboré aux campagnes de terrain Lucas Thénard et Sophie Jarczynka, étudiants de l'UFR de Géographie de l'USTL. Les auteurs remercient MM. Jean-Claude Thouret et Yannick Lageat ainsi que deux relecteurs anonymes dont les critiques et les commentaires constructifs ont permis d'améliorer le manuscrit original.

\section{Références}

Albinet V. (2000) - Géomorphologie et gestion des falaises sur les côtes françaises de l'Atlantique et de la Manche. Thèse, Université de Lyon 2, 661 p.

Alveirinho Dias J. M., Neal W.J. (1992) - Sea cliff retrcat in southern Portugal: profiles, processes and problems. Journal of Coastal Research, 8, 3, 641-654.

Battiau-Queney Y., Simao B., Tékin M. (2003) - Les falaises du Nord-Boulonnais, du cap Gris-Nez à Audresselles (France). Hommes et Terres du Nord, 1, 12-22.

Benumof B.T., Storlazzi C.D., Seymour R.J., Griggs G.B., (2000) - The relationship between incident wave energy and seacliff crosion rate: San Diego County, California. Journal of Coastal Research, 16, 4, 1162-1178.

Bonte A. (1959) - La destruction des falaises du Blanc-Nez. Annales de la Société géologique du Nord, 79, 183-184.

Bracq P., Brunin A.S. (1999) - Approche des relations tectoniquekarst-hydrodynamisme par l'analyse de traçages réalisés dans l'aquifère crayeux du Boulonnais (Escalles, Nord de la France). Geodinamica Acta, 12, 359-370.

Bracq P., Hanich L., Delay F., Crampon N. (1992) - Mise en évidence par traçage d'une relation rapide, entre la surface et les eaux souterraines, liée à des phénomènes de dissolution dans la craic du Boulonnais (Nord de la France). Bulletin de la Société géologique de France, 163, 2, 195-203.

Briquet A. (1930) - Le littoral du Nord de la France et son évolution morphologique. Éd. A. Colin, Paris, 349 p.

Brunsden D., Jones D.K.C. (1976) - The evolution of landslides slopes in Dorset. Philosophical Transactions of the Royal Society of London. Sér. A, 283, 605-631.

Catalao J., Catita C., Miranda J., Dias J. (2000) - Aplicaçāo de técnicas fotogramétricas na medição de taxas de recuo de arribas do Algave (Olhos de Ágau-Quarteira). Ingenium, 46, 82-86.

Catalao J., Catita C., Miranda J., Dias J. (2002) - Photogrammetric analysis of the coastal erosion in the Algarve (Portugal). Géomorphologie : relief, processus, environnement, 2, 119-126.

Clique P.M., Lepetit J.P. (1986) - Catalogue sédimentologique des côtes françaises : côtes de la mer du Nord et de la Manche. Éd. Eyrolles, Paris, 404 p.

Colbeaux J.P., Dupuis C., Robaszinski F., Auffret J.P., Haesaerts P., Sommé J. (1980) - Le détroit du Pas-de-Calais : un élément dans la tectonique de blocs de l'Europe nord-occidentale. Bulletin d'Information des (iéologues du Bassin de Paris, 17, 4, 41-54.

Costa S., Freiré-Diaz S., Di-Nocerra L. (2001) - Le littoral hautnormand et picard : une gestion concertée. Annales de Géographie, 618, 117-135. 
Costa S., Delahaye D., Freiré-Diaz S., Davidson R., Laignel B., Di-Nocerra L. (2002) - Quantification par analyse photogrammétrique du recul des falaises et des apports en galets corrélatifs (Haute-Normandie, France). In : Delahaye D., Levoy F., Maquaire O. (éds). Geomorphology: from expert opinion to modelling, CERG, Strasbourg, 205-214.

Costa S., Lageat Y., Hénaff A., Delahaye D., Plessis E. (2003) Origine de la variabilité spatiale du recul des falaises crayeuses du nord-ouest du Bassin de Paris. L'exemple du littoral haut-normand (France). Hommes et Terres du Nord, 1, 22-31.

Davies P., Williams A.T., Bomboe P. (1998) - Numerical analysis of coastal cliff failure along the Pembrokeshire coast National Park, Wales, UK. Earth Surface Processes and Landforms, 23, 1123-1134.

Debrabant P., Adida B., Painset J., Deconinck J.-F., Recourt P. (1994) - Comportement géotechnique des argiles de Châtillon (Kimméridgien / Tithonien du Bas-Boulonnais). Annales de la Société géologique du Nord, 3 (2 "sćric), 145-153.

Destombes J.P., Destombes P. (1963) - L'anticlinal des Quénocs, au cap Blanc-Nez (Pas-de-Calais). Annales de la Société géologique du Nord, 83, 47-56.

Guilcher A. (1966) - Les grandes falaises et mégafalaises des côtes sud-ouest et ouest de l'Irlande. Annales de Géographie, 407, 26-38.

Hénaff A., Lageat Y, Costa S., Plessis E. (2002) - Le recul des falaises crayeuses du Pays de Caux : détermination des processus d'érosion et quantification des rythmes d'évolution. Géomorphologie : relief, processus, environnement, 2, 107-118.

Hutchinson J.N. (1969) - A reconsideration of the coastal landslides at Folkestone Warren, Kent. Géotechnique, 19, 1, 6-38.

Hutchinson J.N. (1983) - A pattern in the incidence of major coastal mudslides. Earth Surface Processes and Landforms, 8, 391-398.

Hutchinson J.N., Bromhead E.N., Lupini J.F. (1980) - Additional observations on the Folkestone Warren landslides. Quarterly Journal of Engineering of Geology, 13, 1-31.

Jones D. G., Williams A. T. (1991) - Statistical analysis of factors influencing coastal erosion along a section of the west Wales coast, UK. Earth Surface Processes and Landforms, 23, 1123 1134.

Kirkgöz M.S. (1995) - Breaking wave impact on vertical sloping coastal structures. Ocean Engineering, 22, 1, 35-48.

Lahousse P., Pierre G. (2002) - Le recul des falaises crayeuses du Blanc-Nez (Pas-de-Calais, France). In : Delahaye D., Levoy F., Maquaire O. (éds). Geomorphology: from expert opinion to modelling, CERG, Strasbourg, 235-242.

Lahousse P., Pierre G. (2003a) - The retreat of chalk cliffs at cape Blanc-Nez (France): autopsy of an erosional crisis. Journal of Coastal Research, 19, 2, 431-440.

Lahousse P., Pierre G. (2003b) - Vitesse, rythme et modalités du recul des falaises crayeuses du cap Blanc-Nez (Pas-de-Calais, France). Zeitschrift für (Geomorphologie, 47, 2, 215-234.
Lahousse P., Pierre G., Lanoy-Ratel P. (2000) - Quantification par analyse photogrammétrique du recul des falaises du BlancNez depuis 1944 (France, Pas-de-Calais). Photo-Interprétation, 3/4, 3-9, 3 planches.

Leroux B. (1959) - La destruction des falaises kimméridgiennes du Boulonnais. Annales de la Société géologique du Nord, 79, 168-177.

Maquaire O., Gigot P., Ballais, J.L. (1984) - Étude du glissement de Bouffay (5 août 1981) : réflexions en vue de la prévision et de la prévention. In : J.C. Flageollet (dir.) Mouvements de terrains. Document du BRGM, 83, 29-40.

Maquaire O. (1990) - Les mouvements de terrain de la côte du Calvados. Document du BRGM, 197, 431 p.

Moore L.J. (2000) - Shoreline mapping techniques. Journal of Coastal Research, 1, 111-123.

Osman C. W. (1917) - The landslips of Folkestone Warren and thickness of the Lower Chalk and Gault near Dover. Proceedings of the Geologists' Association, 28, 59-84.

Overton M., Petrina C., Fisher J. (1996) - Determining shoreline position using historical photography and digital softcopy photogrammetry, ASPRS/ACSM Annual Convention and Exposition. Technical Paper, 1, 512-513.

Pierre G., Lahousse P. (2003) - Méthodes de quantification du recul des falaises : l'exemple du cap Blanc-Nez (Pas-de-Calais, France). Hommes et Terres du Nord, 1, 32-43.

Prêcheur C. (1960) - Le littoral de la Manche de Sainte-Adresse à Ault. Étude morphologique. Norois, hors-séric, $138 \mathrm{p}$.

Robaszynski F., Amédro F. (1993) - Les falaises crétacées du Boulonnais. La coupe de référence du Cap Blanc-Nez dans un contexte sédimentaire global. Annales de la Société géologique du Nord, 2, 31-44.

Robinson L.A. (1977) - Marine crosive processes at the cliff foot. Marine Geology, 23, 257-271.

Sommé J., Antoine P., Cunat-Bogé N., Lefèvre D., Munaut A.V. (1999) - Le Pléistocène moyen marin de la mer du Nord en France : falaise de Sangatte et formation d'Herzecle. Quaternaire, 10, (2-3), 151-160.

Steers J. A. (1962) - Coastal cliffs: report of a symposium. The Geographical Journal, 128, 3, 303-320.

Sunamura T. (1982) - A predictive model for wave-induced cliff crosion, with application to the pacific coast of Japan. Journal of Geology, 90, 167-178.

Sunamura T. (1992) - Geomorphology of rocky coasts. John Wiley \& Sons, $302 \mathrm{p}$.

Wissocq L. (1992) - Impact géomorphologique des tempêtes de 1989-1990 sur le littoral du cap Blanc-Nez (Pas-de-Calais). Hommes et Terres du Nord, 3, 132-135.

Wood A. M. M. (1955) - Folkestone Warren landslips: investigations, 1948-50. Proceedings of the Institution of Civil Engineers, Railway Paper $n^{\circ}$ 56, 460-464.

Article reçu le 5 janvier 2004, accepté le 6 mai 2004 